\title{
The Drainage of Thin Liquid Films on an Inclined Solid Surface
}

\author{
Joseph G. Abdulahad ${ }^{1}$, Salih A. Derbaz ${ }^{2}$ \\ ${ }^{1,2}$ Department of Mathematics, Faculty of Science, University of Zakho,Duhok, Kurdistan Region, Iraq
}

\begin{abstract}
In this paper, we consider the thinning process of an inclined thin liquid film over a solid boundary with an inclination angle $\boldsymbol{\theta}$ to the horizontal in gravity driven flow. Throughout this work, we assumed that the fluid thickness is constant far behind the front and we neglect the thickness of the film at the beginning of the motion. The equation of the film thickness is obtained analytically, using the similarity method by which we can isolate the explicit time dependence and then the shape of the film will depend on one variable only. The solution of the governing equations of the film thickness is obtained numerically by the Rung-Kutta method with the aid of Mat lab(ode45).
\end{abstract}

Keywords: Thin Liquid Films, Navier-Stokes equations, continuity equation .

\section{INTRODUCTION}

We present here some of the theoretical aspects of the instability development in an inclined thin liquid films on a solid surface in two dimensional coordinate system with an inclination angle $\boldsymbol{\theta}$ to the horizontal . There are different types of phenomena that can occur, such as drainage, details of rupture, non-Newtonian surface properties in moving contract lines in thin liquid films [1]. These phenomena can help to describe the physical processes that occur in our real world. [2,3] have studied the case of contact line instabilities of thin liquid films but with constant flux configuration and also they considered some global models of a moving contact lines. [4] studied the thin liquid films flowing down the inverted substrate in three dimensional flow. [5] investigated the dynamics of an inclined thin liquid films of variable thickness in steady and unsteady cases and when the film is stationary and uniform. [6] considered the stability of thin liquid films and sessile droplets. The stability of the contact line of thin fluid film flows with constant flux configuration is considered by [7]. [8] considered the spreading of thin liquid films with small surface tension in the case when the flow is unsteady. The Non-linear analysis of creeping flow on the inclined permeable substrate plane subjected to an electric field was considered by [9]. In this paper we investigate the drainage of the inclined thin liquid films where the gravity and other forces such as viscous and surface tension forces have a significant effect on the flow of the film. We use the similarity method by which we can isolate the explicit time dependence and then the shape of the film will depend on one variable only. The solution of the governing equations of the liquid film thickness is obtained numerically.

\section{Governing Equations:}

Let $q=(u, w)$ denotes the fluid velocity, where $u$ and $w$ are the velocity components in $\mathrm{x}$ and $\mathrm{z}$ directions respectively. Let $z=h(x, t)$ be the equation of the inclined thin liquid films as shown in Figure (1) and the flow is in $\mathrm{x}$ direction. The continuity equation is given by:

$\frac{\partial u}{\partial x}+\frac{\partial w}{\partial z}=\mathbf{O}$

and from the incompressibility condition, we have

$w=-z \frac{\partial u}{\partial x}$

and this insures that $\frac{\partial \boldsymbol{u}}{\partial \boldsymbol{x}}$ is a function of $\mathrm{x}$ and $\mathrm{t}$ only.

The Navier-Stokes equations in $X$ and $\mathrm{z}$ directions respectively for an inclined thin liquid film are given by:

$\rho\left(\frac{\partial u}{\partial t}+u \frac{\partial u}{\partial x}+w \frac{\partial u}{\partial z}\right)=-\frac{\partial P}{\partial x}+\mu\left(\frac{\partial^{2} u}{\partial x^{2}}+\frac{\partial^{2} u}{\partial z^{2}}\right)+\rho g \sin \theta$

And

$\rho\left(\frac{\partial w}{\partial t}+u \frac{\partial w}{\partial x}+w \frac{\partial w}{\partial z}\right)=-\frac{\partial P}{\partial z}+\mu\left(\frac{\partial^{2} w}{\partial x^{2}}+\frac{\partial^{2} w}{\partial z^{2}}\right)-\rho g \cos \theta$

where $\rho, \mu$ and $P$ are the density, viscosity of fluid and $P$ the pressure. In lubrication theory the inertia terms can be neglected and the Navier-Stokes equations (3) and (4) become 
$\frac{\partial P}{\partial x}=\mu\left(\frac{\partial^{2} u}{\partial x^{2}}+\frac{\partial^{2} u}{\partial z^{2}}\right)+\rho g \sin \theta$

And $\frac{\partial \boldsymbol{P}}{\partial z}=\mu\left(\frac{\partial^{2} w}{\partial x^{2}}+\frac{\partial^{2} w}{\partial z^{2}}\right)-\rho g \cos \theta$

\section{Boundary Conditions}

The boundary conditions to be imposed on the bounding surfaces are as follows:

\section{The no-slip condition gives}

At $z=0, u=0$

The shear stress condition on the surface vanishes, that is:

At $z=\boldsymbol{h}, \frac{\partial \boldsymbol{u}}{\partial z}=\mathbf{O}$

the normal stress condition on the surface of the film is given by

$\bar{w}=-\sigma k$

Where $\sigma$ is the surface tension and $k$ is the curvature of the surface which is given by

$$
k=\frac{\partial^{2} h}{\partial x^{2}} /\left(\left(1+\left(\frac{\partial h}{\partial x}\right)^{2}\right)^{3 / 2}\right.
$$

The pressure is related to the normal stress by the formula

$\boldsymbol{P}=\bar{w}+2 \mu\left(\frac{\partial w}{\partial z}\right)_{s}$

Now from the continuity equation (1), we have

$$
\left(\frac{\partial w}{\partial z}\right)_{s}=-\left(\frac{\partial u}{\partial x}\right)_{s},
$$

and thus equation (11) is then become

$$
P=\bar{w}-2 \mu\left(\frac{\partial u}{\partial x}\right)_{s}
$$

since $\left(\frac{\partial \boldsymbol{h}}{\partial \boldsymbol{x}}\right)^{2}$ is so small for thin liquid films, thus equation (10) is

then reduces to give $\quad k=\frac{\partial^{2} h}{\partial x^{2}}$,

and equation (13) is then become

$P=-\sigma \frac{\partial^{2} h}{\partial x^{2}}-2 \mu\left(\frac{\partial u}{\partial x}\right)_{s}$

Differentiate equation (14), we get

$\frac{\partial P}{\partial x}=-\sigma \frac{\partial^{3} h}{\partial x^{3}}-2 \mu\left(\frac{\partial^{2} u}{\partial x^{2}}\right)_{s}$.

It is to be noted that $\frac{\partial P}{\partial x}$ is a function of $\mathrm{x}$ and $\mathrm{t}$ only on the surface of the

liquid film $\mathrm{z}=\mathrm{h}(\mathrm{x}, \mathrm{t})$.

Now from the conservation of mass and since the free surface is a stream line, the derivative following the motion ( the material or

the substantial derivative) $\frac{D F}{D t}$ must be vanished on $\mathrm{z}=\mathrm{h}(\mathrm{x}, \mathrm{t})$ and thus, we have

$\frac{D F}{D t}=\frac{\partial F}{\partial t}+u \frac{\partial F}{\partial x}+w \frac{\partial F}{\partial z}=0$

Where

$\mathrm{F}(\mathrm{x}, \mathrm{z}, \mathrm{t})=\mathrm{z}-\mathrm{h}(\mathrm{x}, \mathrm{t})$

from equations (16) and (17), we have

$\frac{\partial \boldsymbol{h}}{\partial \boldsymbol{t}}+\boldsymbol{u} \frac{\partial \boldsymbol{h}}{\partial \boldsymbol{x}}-\boldsymbol{w}=\mathbf{O}$

now substitute equation (2) into equation (18), we obtain

$\frac{\partial \boldsymbol{h}}{\partial t}+\boldsymbol{u} \frac{\partial \boldsymbol{h}}{\partial \boldsymbol{x}}+z \frac{\partial \boldsymbol{u}}{\partial x}=\mathrm{O}$ 
on the surface of the liquid film $\mathrm{z}=\mathrm{h}(\mathrm{x}, \mathrm{t})$, equation (19) then gives

$\frac{\partial \boldsymbol{h}}{\partial \boldsymbol{t}}+\boldsymbol{u} \frac{\partial \boldsymbol{h}}{\partial \boldsymbol{x}}+\boldsymbol{h} \frac{\partial \boldsymbol{u}}{\partial \boldsymbol{x}}=\mathrm{O}$

\section{Dimensional Analysis:}

We now introduce the following non-dimensional variables as follow:

$x=L \bar{x}, \quad z=H \bar{z}, u=U \bar{u}, w=\varepsilon U \bar{w}, \quad P=\frac{M U}{L} \bar{P}$,

$h=H \bar{h}$ and $t=T \bar{t}, \quad \varepsilon=\frac{H}{L}<<1$

where $\mathrm{L}, \mathrm{H}$ and $\mathrm{U}$ are the characteristics.

By using the dimensionless variables the continuity equation (1) then gives

$\frac{\partial \overline{\boldsymbol{u}}}{\partial \overline{\boldsymbol{x}}}+\frac{\partial \overline{\boldsymbol{w}}}{\partial \bar{z}}=\mathbf{O}$

Also equations (14) in non-dimensional variables has the form

$\bar{P}=-\frac{\varepsilon}{C a} \frac{\partial^{2} \bar{h}}{\partial \bar{x}^{2}}-2\left(\frac{\partial \bar{u}}{\partial \bar{x}}\right)_{s}$

Where $C a=\frac{\mu U}{\sigma}$ is the capillary number.

Furthermore the Navier-Stokes equations (5) and (6) in non-dimensional variables are respectively given by

$\frac{\partial \overline{\boldsymbol{P}}}{\partial \bar{x}}=\frac{\partial^{2} \overline{\boldsymbol{u}}}{\partial \overline{\boldsymbol{x}}^{2}}+\frac{2}{\varepsilon^{2}} \frac{\partial^{2} \overline{\boldsymbol{u}}}{\partial \bar{z}^{2}}+\frac{B}{C a} \sin \theta$

And $\frac{\partial \overline{\boldsymbol{P}}}{\partial \bar{z}}=\varepsilon^{2} \frac{\partial^{2} \bar{w}}{\partial \overline{\boldsymbol{x}}^{2}}+\frac{\partial^{2} \overline{\boldsymbol{w}}}{\partial \bar{z}^{2}}-\frac{B}{C a} \cos \theta$

Where $B=\frac{\rho g L^{2}}{\sigma}$ is the bond number.

Also equation (20) has the following non-dimensional form

$\frac{\partial \bar{h}}{\partial \bar{t}}+\frac{1}{s} \overline{\boldsymbol{u}} \frac{\partial \overline{\boldsymbol{h}}}{\partial \overline{\boldsymbol{x}}}+\frac{1}{s} \overline{\boldsymbol{h}} \frac{\partial \overline{\boldsymbol{u}}}{\partial \overline{\boldsymbol{x}}}=\mathrm{O}$

From (23) and on the surface of the film, we have

$\frac{\partial \overline{\boldsymbol{P}}}{\partial \overline{\boldsymbol{x}}}=-\frac{\varepsilon}{\boldsymbol{C a}} \frac{\partial^{3} \overline{\boldsymbol{h}}}{\partial \overline{\boldsymbol{x}}^{3}}-2\left(\frac{\partial^{2} \overline{\boldsymbol{u}}}{\partial \overline{\boldsymbol{x}}^{2}}\right)_{s}$

From equations (24) and (27), we get

$\frac{\partial^{2} \bar{u}}{\partial \overline{\boldsymbol{x}}^{2}}+\frac{2}{\varepsilon^{2}} \frac{\partial^{2} \overline{\boldsymbol{u}}}{\partial \bar{z}^{2}}+\frac{B}{C a} \sin \theta=-\frac{\varepsilon}{C a} \frac{\partial^{3} \bar{h}}{\partial \overline{\boldsymbol{x}}^{3}}-2\left(\frac{\partial^{2} \overline{\boldsymbol{u}}}{\partial \overline{\boldsymbol{x}}^{2}}\right)_{s}$

Or $\quad \frac{\varepsilon}{C a} \frac{\partial^{3} \bar{h}}{\partial \bar{x}^{3}}-+3\left(\frac{\partial^{2} \bar{u}}{\partial \bar{x}^{2}}\right)_{s}+\frac{2}{\varepsilon^{2}} \frac{\partial^{2} \bar{u}}{\partial \bar{z}^{2}}+\frac{B}{C a} \sin \theta=0$

Integrating equation (29) with respect to $\bar{z}$, we get

$\left(\frac{\varepsilon}{C a} \frac{\partial^{3} \bar{h}}{\partial \bar{x}^{3}}+3\left(\frac{\partial^{2} \bar{u}}{\partial \bar{x}^{2}}\right)_{s}+\frac{B}{C a} \sin \theta\right) \bar{z}+\frac{2}{\varepsilon^{2}} \frac{\partial \bar{u}}{\partial \bar{z}}=A_{1}(x, t)$

The boundary conditions (7) and (8) in non- dimensional variables have the form:

At $\bar{z}=\mathrm{O}, \overline{\boldsymbol{u}}=\mathrm{O}$

At $\bar{z}=\bar{h}(\bar{x}, \bar{t}) \quad, \quad \frac{\partial \bar{u}}{\partial \bar{z}}=0$

By using the boundary condition (32), equation (30) reduces to give

$$
\left(\frac{\varepsilon}{C a} \frac{\partial^{3} \bar{h}}{\partial \bar{x}^{3}}+3\left(\frac{\partial^{2} \bar{u}}{\partial \bar{x}^{2}}\right)_{s}+\frac{B}{C a} \sin \theta\right) \bar{h}=A_{1}(x, t)
$$

And thus equation (30), gives

$$
\left(\frac{\varepsilon}{C a} \frac{\partial^{3} \bar{h}}{\partial \bar{x}^{3}}+3\left(\frac{\partial^{2} \bar{u}}{\partial \bar{x}^{2}}\right)_{s}+\frac{B}{C a} \sin \theta\right)(\bar{z}-\bar{h})+\frac{2}{\varepsilon^{2}} \frac{\partial \bar{u}}{\partial \bar{z}}=0
$$

Integrating equation (34), we get 


$$
\left(\frac{\varepsilon}{C a} \frac{\partial^{3} \bar{h}}{\partial \bar{x}^{3}}+3\left(\frac{\partial^{2} \bar{u}}{\partial \bar{x}^{2}}\right)_{s}+\frac{B}{C a} \sin \theta\right)\left(\frac{\bar{z}^{2}}{2}-\bar{h} \bar{z}\right)+\frac{2}{\varepsilon^{2}} \bar{u}=A_{2}(x, t)
$$

From the boundary condition (31) and equation (35), we have

$$
A_{2}(\bar{x}, t)=0 \text {, }
$$

And thus equation (35), then gives

$$
\left(\frac{\varepsilon}{C a} \frac{\partial^{3} \bar{h}}{\partial \bar{x}^{3}}+3\left(\frac{\partial^{2} \bar{u}}{\partial \bar{x}^{2}}\right)_{s}+\frac{B}{C a} \sin \theta\right)\left(\frac{\bar{z}^{2}}{2}-\bar{h} \bar{z}\right)+\frac{2}{\varepsilon^{2}} \bar{u}=0
$$

Equation (36) represents the governing equation of an inclined thin liquid film over a solid boundary.

From equation (27), we have

$$
\left(\frac{\partial^{2} \overline{\boldsymbol{u}}}{\partial \overline{\boldsymbol{x}}^{2}}\right)_{s}=\frac{1}{2}\left(\frac{\partial \overline{\boldsymbol{P}}}{\partial \overline{\boldsymbol{x}}}+\frac{\varepsilon}{\mathrm{Ca}} \frac{\partial^{3} \overline{\boldsymbol{h}}}{\partial \overline{\boldsymbol{x}}^{3}}\right)
$$

Also from (25) and (2), we obtain

$$
\frac{\partial \bar{P}}{\partial \bar{z}}=-\varepsilon^{2} \frac{\partial^{3} \bar{u}}{\partial \bar{x}^{3}} \bar{z}-\frac{\beta}{C a} \cos \theta
$$

Since the term in equation (38) $\varepsilon^{2} \frac{\partial^{3} \overline{\boldsymbol{u}}}{\partial \overline{\boldsymbol{x}}^{3}}$ is very small so we can neglected it and equation (38) reduces to give

$$
\frac{\partial \overline{\boldsymbol{P}}}{\partial \bar{z}}=-\frac{B}{C a} \cos \theta
$$

Or $\bar{P}(\bar{x}, \bar{z}, \bar{t})=-\frac{B}{C a} \cos \theta \bar{z}+A_{3}(\bar{x}, \bar{t})$

Assume that at $\bar{z}=\mathrm{O}, \bar{P}=p_{\mathrm{O}}$ and from equation (40) we get $A_{3}(\bar{x}, \bar{t})=p_{0}$ and so equation

(40), then gives $\bar{P}(\bar{x}, \bar{z}, \bar{t})=-\frac{B}{C a} \cos \theta \bar{z}+p_{\mathrm{o}}$

On the surface of the film $\bar{z}=\overline{\boldsymbol{h}}(\overline{\boldsymbol{x}}, \overline{\boldsymbol{t}})$ equation (41), gives

$$
\begin{aligned}
& \overline{\boldsymbol{P}}(\overline{\boldsymbol{x}}, \bar{z}, \overline{\boldsymbol{t}})=-\frac{B}{C a} \cos \theta \overline{\boldsymbol{h}}+p_{\mathrm{o}} \\
& \text { or } \frac{\partial \overline{\boldsymbol{P}}}{\partial \overline{\boldsymbol{x}}}=-\frac{\boldsymbol{B}}{\boldsymbol{C} \boldsymbol{a}} \cos \theta \frac{\partial \overline{\boldsymbol{h}}}{\partial \overline{\boldsymbol{x}}}
\end{aligned}
$$

From equations (37) and (43), we get

$$
\left(\frac{\partial^{2} \bar{u}}{\partial \bar{x}^{2}}\right)_{s}=\frac{B}{2 C a} \cos \theta \frac{\partial \bar{h}}{\partial \bar{x}}-\frac{\varepsilon}{2 C a} \frac{\partial^{3} \bar{h}}{\partial \bar{x}^{3}}
$$

Thus from equations (36) and (44), we obtain

$$
\begin{aligned}
& \left(\frac{\varepsilon}{C a} \frac{\partial^{3} \bar{h}}{\partial \bar{x}^{3}}+\frac{3 B}{2 C a} \cos \theta \frac{\partial \bar{h}}{\partial \bar{x}}-\frac{3 \varepsilon}{2 C a} \frac{\partial^{3} \bar{h}}{\partial \bar{x}^{3}}+\frac{B}{C a} \sin \theta\right)\left(\frac{\bar{z}^{2}}{2}-\bar{h} \bar{z}\right)+\frac{2}{\varepsilon^{2}} \bar{u}=0 \\
& \text { Or }\left(\frac{3 B}{2 C a} \cos \theta \frac{\partial \bar{h}}{\partial \bar{x}}-\frac{\varepsilon}{2 C a} \frac{\partial^{3} \frac{h}{\partial \bar{x}^{3}}}{2 C}+\frac{B}{C a} \sin \theta\right)\left(\frac{\bar{z}^{2}}{2}-\bar{h} \bar{z}\right)+\frac{2}{\varepsilon^{2}} \bar{u}=0 \\
& \text { or } \bar{u}=-\left(\frac{3 \varepsilon^{2} B}{4 C a} \cos \theta \frac{\partial \bar{h}}{\partial \bar{x}}-\frac{\varepsilon^{3}}{4 C a} \frac{\partial^{3} \bar{h}}{\partial \bar{x}^{3}}+\frac{\varepsilon^{2} B}{2 C a} \sin \theta\right)\left(\frac{\bar{z}^{2}}{2}-\bar{h} \bar{z}\right)
\end{aligned}
$$

the second term in equation (45) is very small and so we can neglected and thus equation (45) reduces to give

$$
\bar{u}=-\left(\frac{3 \varepsilon^{2} B}{4 C a} \cos \theta \frac{\partial \bar{h}}{\partial \bar{x}}+\frac{\varepsilon^{2} B}{2 C a} \sin \theta\right)\left(\frac{\bar{z}^{2}}{2}-\bar{h} \bar{z}\right)
$$

The average velocity over the film thickness is given by

$$
\bar{u}_{a v}=\frac{1}{h} \int_{0}^{h} \bar{u} d z
$$

Equations (46) and (47) then give

$\bar{u}_{a v}=\frac{\varepsilon^{2} B}{4 C a} \cos \theta \bar{h}^{2} \frac{\partial \bar{h}}{\partial \bar{x}}+\frac{\varepsilon^{2} B}{6 C a} \sin \theta \bar{h}^{2}$

or from equations (26) and (48), we have 


$$
\begin{aligned}
& \frac{\partial \bar{h}}{\partial \bar{t}}+\frac{1}{S}\left(\frac{\varepsilon^{2} B}{4 C a} \cos \theta \bar{h}^{2} \frac{\partial \bar{h}}{\partial \bar{x}}+\frac{\varepsilon^{2} B}{6 C a} \sin \theta \bar{h}^{2}\right)+\frac{\bar{h}}{S}\left(\frac{\varepsilon^{2} B}{4 C a} \cos \theta \bar{h}^{2} \frac{\partial^{2} \bar{h}}{\partial \bar{x}^{2}}\right. \\
& \left.+\frac{\varepsilon^{2} B}{2 C a} \cos \theta \bar{h}\left(\frac{\partial \bar{h}}{\partial \bar{x}}\right)^{2}+\frac{\varepsilon^{2} B}{3 C a} \sin \theta \bar{h}^{2} \frac{\partial \bar{h}}{\partial \bar{x}}\right)=O
\end{aligned}
$$

After simplifications equation (49) reduces to give

$$
\frac{\partial \bar{h}}{\partial \bar{t}}+\frac{3 \varepsilon^{2} B}{4 C a S} \cos \theta \bar{h}^{2}\left(\frac{\partial \bar{h}}{\partial \bar{x}}\right)^{2}+\frac{\varepsilon^{2} B}{2 C a S} \sin \theta \bar{h}^{2} \frac{\partial \bar{h}}{\partial \bar{x}}+\frac{\varepsilon^{2} B}{4 C a S} \cos \theta \bar{h}^{3} \frac{\partial^{2} \bar{h}}{\partial \bar{x}^{2}}=\mathrm{O}
$$

After simplifications equation (49) reduces to give

\section{Similarity Method}

We concentrate on equation (50) since it represents the flow ofa vertical film down a substrate. We have assumed through this work that the film thickness is constant far behind the front and this assumption is true since the fluid thins out there and so we apply the similarity method to see how this thinning process evolves in time.

for self-similar solution, we assume that

$h(\bar{x}, t)=h_{\circ}(t) \bar{H}(\eta)$

where $\eta$ is the self similar variable measures the distance to the front and

$\eta=\frac{\bar{x}}{\bar{x}_{f}(t)}, \quad 0 \leq \eta \leq 1$

where $\bar{x}_{f}(t)$ is the front position as shown in figure (1), this method is to isolate the explicit time dependence and then the shape of the film will depend on the variable $\eta$ only.

Now let

and

$$
\left.\begin{array}{l}
\boldsymbol{h}_{\circ}(t)=D t^{\alpha} \\
\bar{x}_{f}(t)=A t^{\delta}
\end{array}\right\}
$$

where $\mathrm{D}$ and $\mathrm{A}$ are the self similar exponents.

from (51) and (52), equation (50) then gives

$$
\begin{aligned}
& -D \alpha t^{\delta-1} \eta \frac{d \bar{H}}{d \eta}+D \delta t^{\delta-1} \bar{H}-\frac{3 B \varepsilon^{2} D^{4}}{4 C a S A^{2}} \cos \theta t^{4 \delta-2 \alpha} \bar{H}^{2}\left(\frac{d \bar{H}}{d \eta}\right)^{2+} \\
& \frac{B \varepsilon^{2} D^{3}}{2 C a S A} t^{3 \delta-\alpha} \sin \theta \bar{H}^{2} \frac{d \bar{H}}{d \eta}+\frac{B \varepsilon^{2} D^{4}}{4 C a S A^{2}} \cos \theta t^{4 \delta-2 \alpha} \bar{H}^{3} \frac{d^{2} \bar{H}}{d \bar{x}^{2}}=0
\end{aligned}
$$

By equating the exponents of $\mathrm{t}$ in equation (54), we get

$$
\alpha=\delta=-1
$$

And thus equation (54) reduces to give

$$
\eta \frac{d \bar{H}}{d \eta}-\bar{H}+\frac{3 B \varepsilon^{2} D^{3}}{4 C a S A^{2}} \cos \theta \bar{H}^{2}\left(\frac{d \bar{H}}{d \eta}\right)^{2}+\frac{B \varepsilon^{2} D^{2}}{2 C a S A} \sin \theta \bar{H}^{2}\left(\frac{d \bar{H}}{d \eta}\right)+\frac{B \varepsilon^{2} D^{3}}{4 C a S A^{2}} \cos \theta \bar{H} \frac{d^{2} \bar{H}}{d \eta^{2}}=0
$$

Equation (55) represents the non- linear ordinary differential equation for the thickness of the inclined thin liquid film in $(\eta, \bar{H})$-plane fiqures (2) (3) and (4) show that the thickness of liquid film for all liquids decreases as the time increases and this is usual from fiqure (5) and for liquids such as glycerin, linseed oil and olive oil the solution curves show that as the angle of the inclination angle increases, the thickness of the liquid films increases, while in other liquids such as water ,carbon tetracloride and mercury the case is different, the solution curves show that the thickness of the liquid films increases as the angle of inclination decreases and the reason for this distinction will be discussed later in the conclusion.

\section{Figures And Tables}




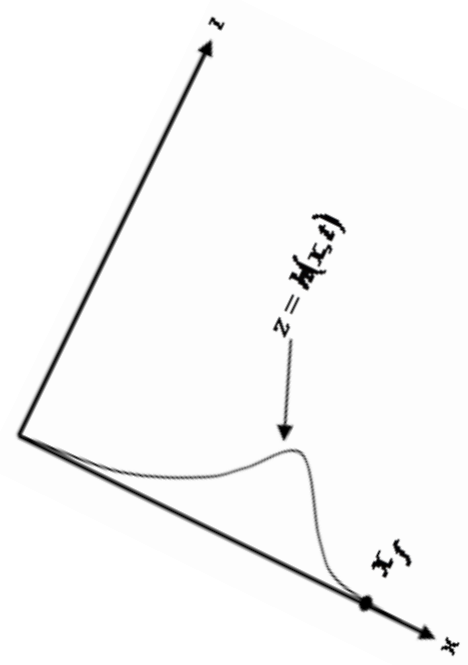

Fig. (1): Sketch of the flow in two-dimensional geometry, where the capillary ridge is just behind the flow front.

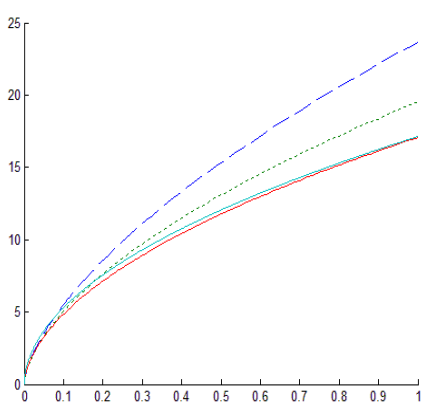

(water)

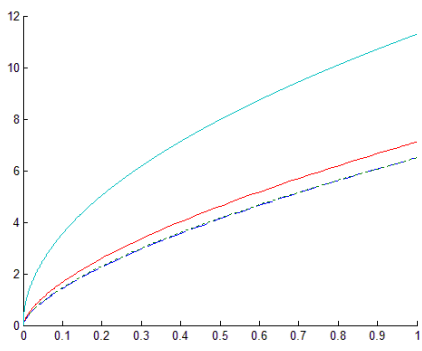

(Linseed oil)

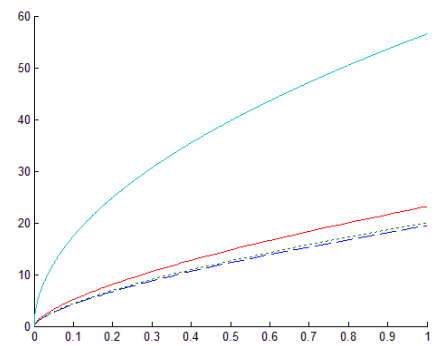

(Glyserin)

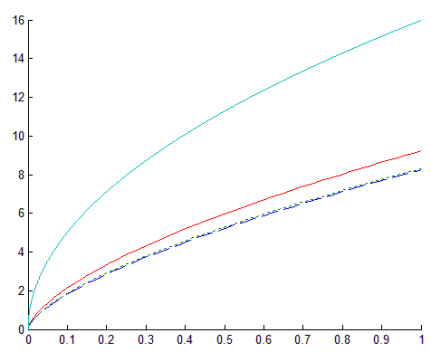

(Olive oil)

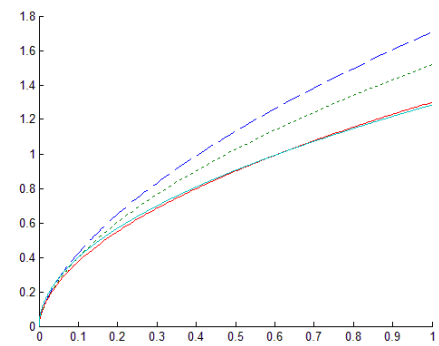

(Carbon Tetrachloride)

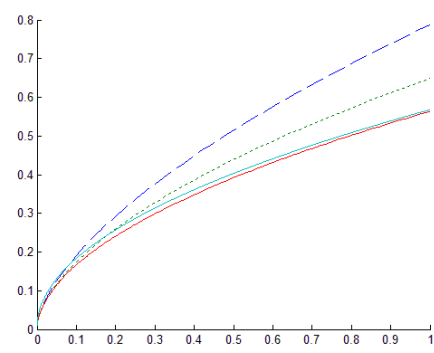

(Mercury)

Fig.(2): Thickness of some thin liquid films for different inclination angles namely $\ldots$ $\theta=0.52-\theta=1.04-\theta=1.57$

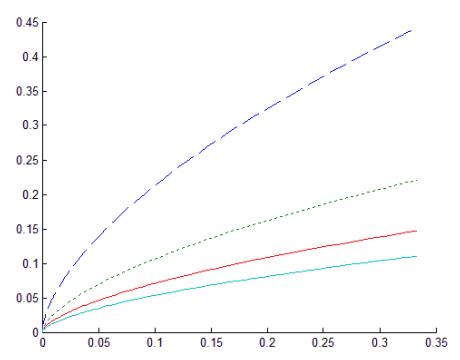

(water)

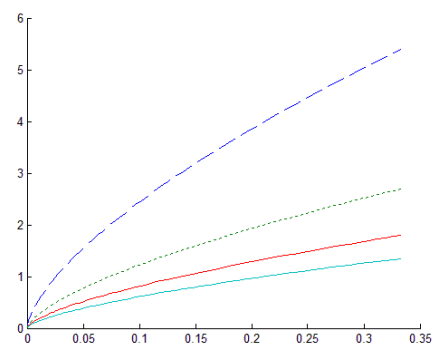

(Glyserin)

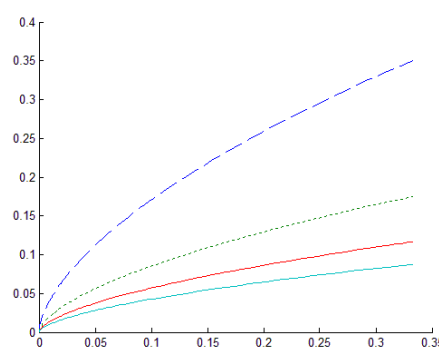

(Carbon Tetrachloride) 


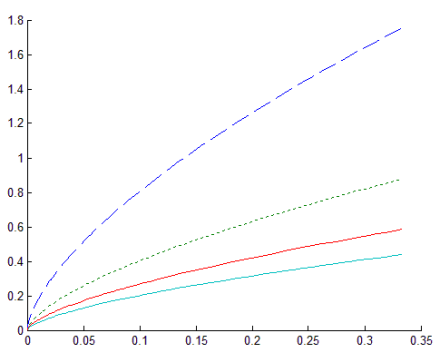

(Linseed oil)

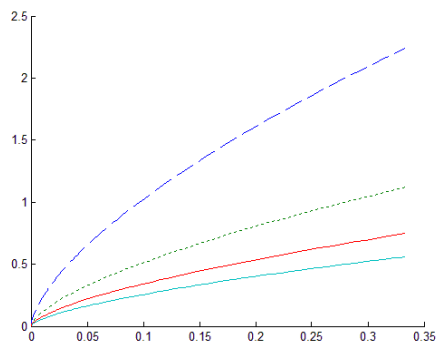

(Olive oil)

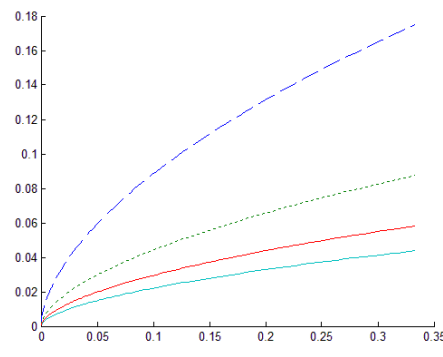

(Mercury)

Fig.(3): Thickness of thin liquid films in $(\bar{x}, \bar{h})$-plane for $\theta=\frac{\pi}{6}$, for different time $\cdots t=1 \quad t=2$ $-\mathrm{t}=3-\mathrm{t}=4$

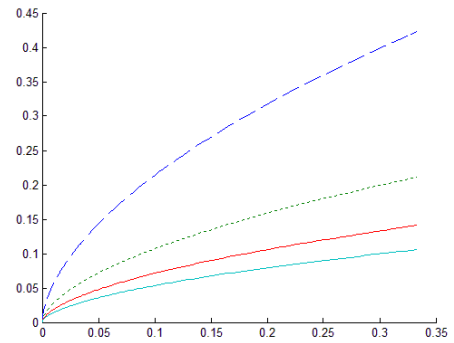

(water)

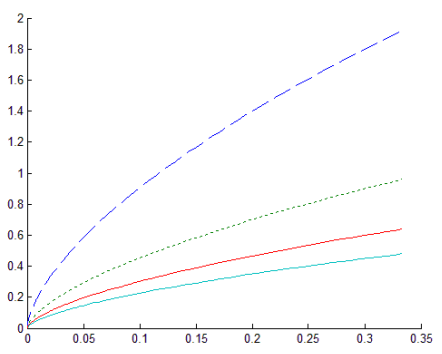

(Linseed oil)

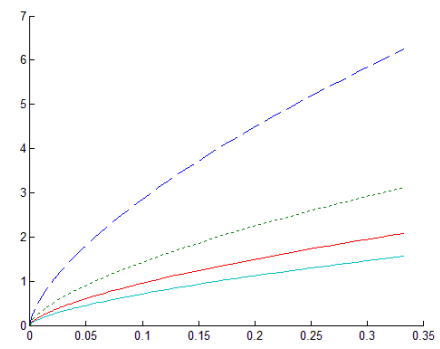

(Glyserin)

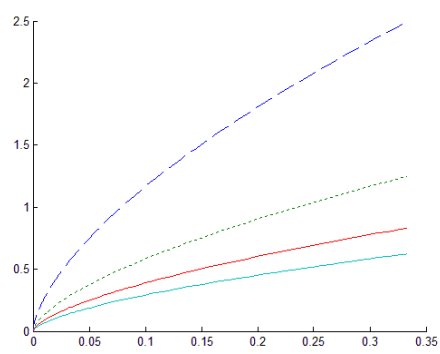

(Olive oil)

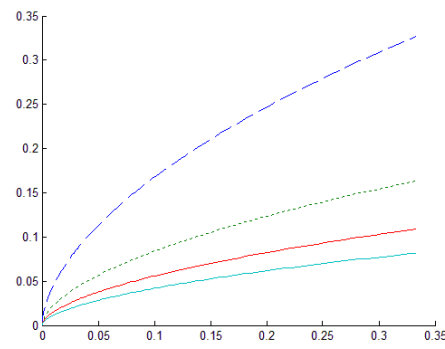

(Carbon Tetrachloride)

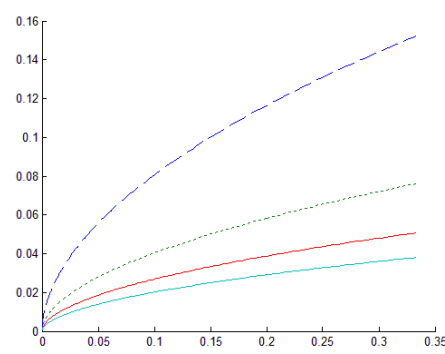

(Mercury)

Fig.(4): Thickness of thin liquid films in $(\bar{x}, \bar{h})$-plane for $\theta=\frac{\pi}{3}$, for different time $\ldots t=1 \quad t=2$ $-\mathrm{t}=3-\mathrm{t}=\mathbf{4}$

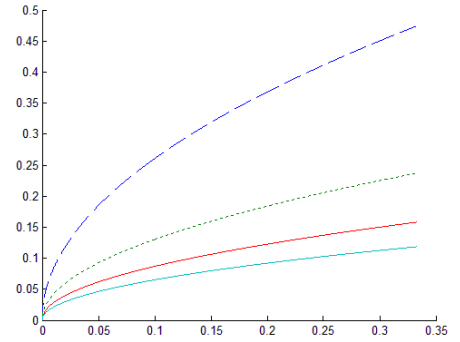

(water)

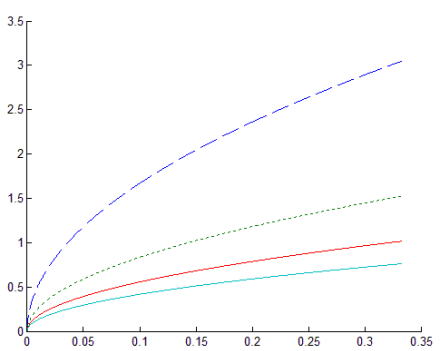

(Linseed oil)

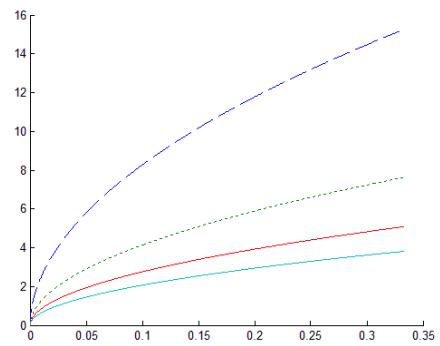

(Glyserin)

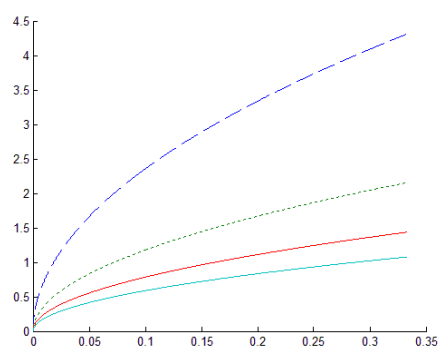

(Olive oil)

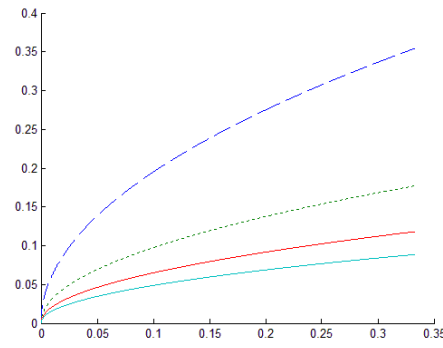

(Carbon Tetrachloride)

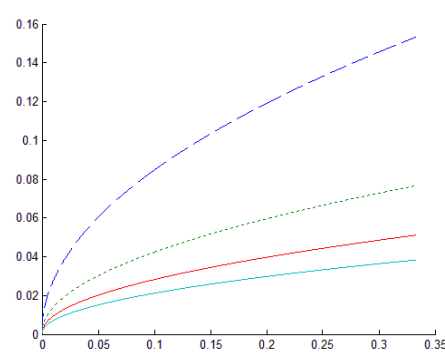

(Mercury)

Fig.(5): Thickness of thin liquid films in $(\bar{x}, \bar{h})$-plane for $\theta=\frac{\pi}{2}$, for different time $\ldots$

$-\mathbf{t}=\mathbf{3}-\mathbf{t}=\mathbf{4}$ 


\section{CONCLUSION}

The dynamicsofafree surface liquid films flowing over an inclined solid boundary with an inclination angle to the horizontal is very useful in industrial coating and spinning processes. The non-linear differential equation that governs such flow for unsteady case in non-dimensional form is obtained. The similarity method is used to solve differential equation for the drainage of an inclined thin liquid film for different liquids namely for water, glycerin, carbon tetrachloride, linseed oil, olive oil, and mercury, this method is very useful since the non-linear partial differential equations that governs such flow can be simplified to a single ordinary differential equation of one variable. The solution curves of the differential equation shows that the thickness of a liquid film increases as the time decreases as the time increases and this is usual.Forthermore, for different liquids such as glycerin, linseed oil, and olive oilthe solution curves show that vas the angle of the inclination angle increases, the thickness ofyhe liquid films increases and the reason for this is related to small values of viscosities for these liquids and large value of the ratio between the Bond number and the capillary number, while in the case of other liquids like water, carbon tetrachloride and mercury the case is different, the solution curves show that the thickness of the liquid films increases as the angle of inclination decreases since the liquids have large viscosities and small vaules of the ratio between the Bond number and the Capillary number.

\section{REFERENCES}

[1] Bertozzi, A.L., The mathematics of moving contact lines in thin liquid film,Amer. Math. Soc.1998, Pp. 689-697, 45.

[2] Diez, J., Kondic L. and Bertozzi, A.L. (2001). Global models for moving contact lines, Phys. Rev., pp. $011208,63$.

[3] Kondic L. and Diez J. (1994). Contact line instabilities of thin fluid film flows: constant flux configuration, Phys. Fluids, pp. 224234.

[4] Lin. T. S., Kondic, L. and Filippove, A.(2012). Thin films flowing down inverted substrated three dimensional flow, Physics of fluids, Vol.24, pp. 1-18.

[5] Karmina, K. A., (2013). Fluid flow and stability analysis in certain thin liquid films.M.Sc.thesis, University of Zakho.

[6] Drofler, F., Rauscher M. and Dietrich S.(2013). Stability of thin liquid films and sessile droplets under confinement,Eur. Phys. J. E.Vol.20, pp. 1-14.

[7] Kondic L (2003). Instabilities in gravity driven flow of thin fluid films, Siam Review, vol. 45, No. 1, pp. 95-115.

[8] Moriarty J.A., and Schwartz L.W.(1991). Unsteady spreading of thin liquid films with small surface tension,American institute of Physics, vol3, No.5, pp. 733-742.

[9] Zakariya, K., Sirweh, M. A. and Alkharashi, S. A.(2012), Non-linear analysis of creeping flow on the inclinedpermeable substrate plane subjected to an electric field. International Journal of Non-linear Mathematics, vol.47, pp. 577-598. 